\title{
SPACE WEATHER IMPACT ON RADIO DEVICE OPERATION
}

\section{O.I. Berngardt}

Institute of Solar-Terrestrial Physics SB RAS,
Irkutsk,Russia,berng@iszf.irk.ru

\begin{abstract}
This paper reviews the space weather impact on operation of radio devices. The review is based on recently published papers, books, and strategic scientific plans of space weather investigations. The main attention is paid to ionospheric effects on propagation of radiowaves, basically short ones. Some examples of such effects are based on 2012-2016 ISTP SB RAS EKB radar data: attenuation of ground backscatter signals during solar flares, effects of traveling ionospheric
\end{abstract}

disturbances of different scales in ground backscatter signals, effects of magnetospheric waves in ionospheric scatter signals.

Keywords: space weather, technical effects.

\section{INTRODUCTION}

The problems of space weather impact on the developed technological society, and in particular on operation of radio electronic devices, have recently become especially acute. In connection with the inclusion of computer and robotic technologies in most of our daily life, a natural question arises: how steadily and correctly this electronic equipment (not always controlled by ordinary users) and its software can operate under varying external conditions [Goodman, Aarons, 1990].

The problem arose long ago owing to interference effects in wire systems [Barlow, 1849] and failures in electric power transmission networks [Love, Coïsson, 2016], especially strong at high latitudes.

Nowadays, a sharp increase is being observed in the number of high precision equipment, which sometimes has inconspicuous peculiar properties insignificant under normal conditions. However, under conditions that are different from those expected, such peculiar properties may be critical for functioning of radio electronic devices, including commonly used household appliances [Whiteson et al., 2014].

The problem of space weather impact on radio devices for regular users has become most evident from the analysis of data acquired with global positioning systems, which are currently the de facto basic element of positioning and timing systems. The main function of this system - the precise positioning - turns out to depend on characteristics of a medium. In particular, during geomagnetic disturbances, the systems can go wrong more often and more seriously and sometimes even fail [Afraimovich et al., 2004; Afraimovich et al., 2007; Kim et al., 2014]. This effect manifests itself in positioning of both terrestrial and space objects [Xiong et al., 2016].

Sudden space weather disturbances leading to powerful scattered signals in radars, radio communications, and radiosondes [Bagaryatsky, 1961; Sverdlov, 1982], require developing systems for predicting such interference and reducing the degree of its influence on radio devices.

Thus, the assessment of space weather impact on operation of radio devices, the forecast of its consequences, the readiness for the problems caused by this impact, and the elimination of its possible effects are the urgent tasks facing any technologically advanced society [The Sun to the Earth - and Beyond ..., 2003; Solar and Space Physics ..., 2013]. The interval between putting equipment into operation, emergence of operational problems, construction and putting into operation of new, more reliable equipment in many cases comprises several years. These intervals are especially long for space-based equipment. A natural solution to this problem will be to take into account the possibility of failure and to estimate space weather effects on the final result of operation of this equipment before it is replaced with a new one, as well as to predict possible failure periods.

The problem of assessing the space weather impact on different areas of human activity and reducing its consequences is usually solved in a variety of ways from implementation of national strategies [Solar and Space Physics ..., 2013; National Space Weather Strategy, 2015], plans [National Space Weather Action Plan, 2015], legislative acts [Obama, 2016], and available information systems [Space Weather - Effects on Technology, 2012] to the participation of enthusiasts and the use of capabilities of household devices and computers (the so-called citizen science [Barnard et al., 2014, Aurorasourus, 2016; Wikipedia, 2016]). Various monitoring and forecasting systems, both global [http://www.swpc. noaa.gov/] and local ones designed for specific aspects of space weather [Love et al., 2016] are stimulated and supported. In-depth reviews of space weather effects on equipment of different types can be found in the monographs [The Sun to the Earth ..., 2003; 
Solar and Space Physics ..., 2013; Effects of Space Weather ..., 2004; Goodman, 2005; Space Weather ..., 2007].

The main geo-effective space weather effects extensively studied today include [National Space Weather Strategy, 2015]: solar radio bursts affecting the operation of radio receivers; induced geoelectric fields influencing wired energy supply and communication systems; ionizing radiation affecting the efficiency of electronic equipment and the vital activity of organisms; expansion of upper atmospheric layers leading to an increase in temperature and density of these layers and affecting the dynamics and lifetime of artificial Earth satellites; as well as ionospheric disturbances affecting radio wave propagation and scattering.

The launch of the system of impulse decameter coherent radars at ISTP SB RAS, in particular under the project "National Heliogeophysical Complex of the Russian Academy of Sciences", raises questions of continuous space weather monitoring for solving not only fundamental but also applied problems important for a technologically advanced society.

\section{ISSUES OF SPACE WEATHER FORMATION}

The term "severe space weather" has arisen relatively recently to describe the influence of solar and geomagnetic activity on equipment operation and infrastructure performance [Severe Space Weather Events, 2008; Solar and Space Physics, 2013], although space weather effects have long been known [Barlow, 1849]. A decisive influence on the major part of large-scale terrestrial phenomena is exercised by the source of radiation and particles closest to Earth - the Sun. Although there are examples of the feedback effect of human activity on large-scale natural processes and the artificial generation of some natural phenomena [Baker et al., 2014; Gombosi et al., 2017], but the Sun can be considered nowadays as the main and permanent source of space weather formation. The particle and radiation flux from the Sun, highly dynamic in space and time, is associated with internal solar processes, and, since it is impossible to monitor deep processes on the Sun in real time, the flux could only be averagely predicted. A human acts mainly as an observer of solar activity variations and as a researcher of regular processes occurring in the upper layers of the Sun. The 11-12-year solar activity cycles, which are related to reversals of the solar magnetic field and reveal themselves in all its parameters from radio emission (F10.7) to the number of sunspots (Wolf number) optically observed for several centuries, are well-known. The axial rotation of the Sun with a period 25-30 days also cause periodic variations in particle-wave radiation fluxes.

The propagation velocity of such fluxes from the Sun to Earth varies: wave radiation propagates at the speed of light and reaches Earth in about 8 minutes, the corpuscular component moves approximately thousand times slower. Thus, if in the first case the motion of radiation in most problems may be considered to be straight and the motion of objects in the solar system may be ignored, for the particle motion we should take into account the Sun rotation, the orbital motion of Earth and its daily rotation, and calculate the particle motion in the resulting complex trajectory in terms of geometry (cone) of particle release from the Sun and distribution of their velocities. Therefore, it is difficult to assess solar particle-wave flux effects, in view of possible delays in appearence of these effects, their extension in time and space (due to the difference in particle velocities and angles of their release from the Sun) [Odstrcil, 2003], as well as the possibility of particle accumulation in Earth's magnetosphere (which leads to additional time delays of the effects). Solving this problem requires creating complex physical models, including numerical, large computational resources, long-term and accurate experiments, as well as a large set of diverse diagnostic instruments at various points of Earth and outer space, which operate in the mode of continuous monitoring and data transfer to centers of their storage, automatic processing, and real-time cosimulation. An essential contribution to the solution of this problem is measurements of these fluxes by the $\mathrm{ACE}$ and DSCVR satellites at the Lagrange point L1, at a distance of about 1 million $\mathrm{km}$ from Earth. They allow us to increase the accuracy and to detail the short-term predictions of composition and dynamics of particle-wave radiation [Machol et al., 2012] as compared to more forehand, albeit less accurate and detailed predictions based on remote observations of solar activity with various (ground- and space-based) telescopes.

Particle radiation, reaching the boundary of Earth's magnetosphere, interacts with it. Trajectories of charged particles bend significantly, and the particles begin to move under strong influence of the geomagnetic field, generating electric fields and currents in the magnetosphere.

An important role here is played by high-latitude regions around magnetic poles (cusps), where the direction of the magnetic field is close to vertical. This causes charged particles to precipitate from the magnetosphere into this region toward Earth's surface. The observed optical effects - aurora borealis - have been well known for a long time and represent one of the consequences of the arrival of solar disturbance in Earth's magnetosphere. Regular observations of similar effects have also been conducted since the last century and carried out by scientists with various special-purpose optical instruments such as all-sky cameras, photometers, as well as by amateur photographers.

Precipitating particles cause a change in ionizationrecombination processes in the lower layers of the ionosphere (D and E) and, in turn, increase the electron density there. This increase leads to an increase in radio wave absorption, which manifests itself as an amplitude decrease or the loss of radio signals on the paths passing through these regions.

Such effects are monitored by observing the amplitude of radio signals over long radio paths (e.g., with 
networks of inclined sounding ionosondes or by receiving a signal from broadcasting stations) or the amplitude of radio signals from space sources (e.g., with riometers). A decrease in signal amplitude is also one of the consequences of the arrival of particles and radiation in Earth's magnetosphere.

The voltage difference arising from separation of charges moving in the solar wind in Earth's magnetosphere under the influence of the magnetic field brings about the formation of field-aligned (along magnetic field lines) currents. These currents close through the $\mathrm{E}$ layer (90-120 km above Earth's surface), which has a maximum electrical conductivity due to peculiarities of distributions of collision frequencies of charged and neutral particles. The strong horizontal currents forming in the E layer of the polar ionosphere generate magnetic field disturbances recorded on Earth's surface with instruments for measuring the full magnetic field vector magnetometers. These geomagnetic disturbances, observed since the nineteenth century, are also one of the consequences of the arrival of charged particles in Earth's magnetosphere.

Besides the processes caused by the motion of charged particles in the ionosphere, the geomagnetic field structure changes due to the appearance of additional charged sources. The main manifestation of this effect, associated with the regular particle-wave radiation of the Sun (solar wind), is, of course, the difference between the external geomagnetic field and the dipole field, including the existence of a sunward sharp flat transition region and an antisunward strongly elongated region.

The solar wind can change the size and shape of the magnetosphere. As a result, the geographic area of the phenomena emerging from particle precipitation (auroras borealis, strong ionospheric currents, radio wave absorption) shifts from high to middle latitudes. In this case, we can observe, say, auroras borealis at latitudes of central regions and southern borders of the Russian Federation (up to the 40 degree magnetic latitude), where it is usually not observed [Feldshtein et al., 2010]. The remaining effects (radio wave absorption, strong currents in the ionosphere, and geomagnetic disturbances) demonstrate similar dynamics during intensification of solar wind fluxes.

It is obvious that solar radiation comes much earlier than corpuscular radiation and also influences processes occurring in Earth's upper atmosphere. The main effect is the very existence of the ionosphere - a plasma layer ionized by solar radiation in Earth's neutral atmosphere. Accordingly, any variations in solar radiation cause variations in the ionospheric electron density at heights corresponding to the lines of radiation absorption by gases constituting Earth's atmosphere and ionosphere. Thus, electron density variations at different heights above Earth's surface [Mikhailov, Perrone, 2016] may be one of the consequences of solar wind disturbances.

An important fact is the existence of well separated zones in Earth's ionosphere, magnetosphere, and atmosphere, on the boundary of which characteristics of the medium change drastically. This leads to the existence of eigenoscillations in these zones, which propagate in the medium relatively independently.

Such oscillations may appear as Schumann resonances in the layer between Earth's surface and ionosphere [Schumann, 1952], internal gravity waves in the atmosphere and their effects in the ionosphere [Lognonné et al., 1998], fast magnetosonic waves in the magnetosphere [Leonovich, Mazur, 2008], and so on. Therefore, many processes occurring in the magnetosphere-ionosphere-atmosphere system can be considered as a superposition of eigenoscillations of this system. Those oscillations that least fade out with time exist the longest in this system and produce aftereffects when the system continues to change despite that the cause of these changes has already disappeared.

This system is also characterized by dynamics under the driving force. For example, gravity variations due to the periodic motion of the Moon around Earth lead to the formation of tidal waves, which have an effect not only on the ocean, but also on the atmosphere and ionosphere [Alpert, 1949]; and the motion of the day-night boundary (solar terminator) in the atmosphere, to the formation of internal gravity waves.

Thus, Earth's magnetosphere, ionosphere, and atmosphere have both eigen and forced oscillations, which can lead to the formation of additional disturbances during the periods when the solar wind effect is absent or has already disappeared. This sometimes causes an additional solar wind effect in time and space, including the appearance of the "memory" effect in the magnetosphere-ionosphere-atmosphere system.

\section{SPACE WEATHER IMPACT ON RADIO DEVICES}

The operation of radio devices depends on a combination of electromagnetic processes inside and outside the devices. We call effects direct if a malfunction is caused by processes inside a radio device, and indirect if it occurs outside the device.

Direct effects involve the induction of electromagnetic fields in conductors inside a radio device, a change in potentials due to additional ionization by background radiation, the emergence of auxiliary currents due to penetration of additional charges from outside, as well as an increase in the background electromagnetic radiation of various types and concentration of different particles during disturbances. This can cause radio equipment malfunctions under the influence of induced current, which leads to hardware and software malfunctions, a decrease in the signal-to-noise ratio, additional ionization of the equipment by electromagnetic radiation, and particleinduced changes in equipment characteristics.

Indirect effects include changes in the medium of radio signal propagation, such as a change in the refractive index of the ionosphere. In this case, the malfunction of the equipment is associated with a change in the medium it uses to transfer data or operate. Depending on types of medium, indirect effects can be classified as changes of conditions in the magnetosphere, ionosphere, and atmosphere, on or under Earth's surface. 


\section{Solar radio bursts}

The most intense solar effect is the electromagnetic radiation observable in various parts of the solar spectrum. Solar radio noise and radio bursts (sudden enhancements of radio emission), discovered in the 1940s, have been quite actively investigated to this day [Bastian et al., 1998; Solar and S pace W eather R adiophysics ..., 2004; Lee, 2007; Shibasaki et al., 2011].

In addition to the general substantial increase in the level of radio emission, variations in the intensity are possible within the radio burst with periods from milliseconds to seconds [Chernov, 2011]. This leads to an additional increase in the instantaneous intensity of radio emission by tens of decibels compared to the average level of the burst [Benz, 1986], which is already higher than the level of the quiet-Sun radio emission. Due to these features, the main effect of radio bursts is reduced to the occurrence of unexpected interference in radar, radio communication, and radio reception devices [Bala et al., 2002].

\section{Induced geoelectric fields}

Geomagnetic disturbances can cause an amplification of currents in the earth's crust, mainly due to the amplification of auroral currents in the polar ionosphere [Boteler, 1994; Pirjola, 2000]. Geomagnetically induced currents affect the stable operation of electric systems [Campbell, 1978; Pulkkinen et al., 2005; Thomson et al., 2011]. As such, they are direct mechanisms of influence on radio electronic devices. These currents are probably the first observable manifestation of the space weather effect on electrical devices [Barlow, 1849].
At present, some organizations use geoelectric field forecasting systems [Erinmez et al., 2002; Thomson et al., 2011]. The increasing interest in geoelectric fields is associated with effects in electric power networks. These effects often cause long-term malfunctions in the networks in North America, Sweden, and Australia [Béland, Small, 2004; Pulkkinen et al., 2005; Marshall et al., 2011].

\section{Ionizing radiation}

The effect of radiation on various electronic devices has been known for quite a long time [Ionizing Radiation Effects ..., 2015]; it reduces to a change in characteristics (constant and temporary) of the devices due to incoming radiation or its related atmospheric processes. This effect can be observed even on consumer devices, such as smartphones [Whiteson et al., 2014].

These effects are most severe in space vehicles. On average, according to CLUSTER data, a solar-radiationinduced decrease in the efficiency of solar panels on board satellites is about $5 \%$ per year. This limits the time of their operation [Keil, 2007]. An even more important effect is the degradation of optical and electronic equipment on board satellites, which may also lead to their loss [Lotóaniu et al., 2015].

Currently, more than 8000 flights a year pass over the North Pole [Space Weather - Effects on Technology, 2012], hence the need to take into account the effect of solar radiation on health of flight personnel, passengers, and on electronic equipment.

Modes of radio wave propagation in the ionosphere at different frequencies

\begin{tabular}{|c|c|c|}
\hline Range & Frequencies & Propagation mode \\
\hline ULF & $<3 \mathrm{kHz}$ & Waveguide, surface wave \\
\hline VLF & $3-30 \mathrm{kHz}$ & Waveguide, surface wave \\
\hline LF (LW) & $30-300 \mathrm{kHz}$ & Waveguide, surface wave \\
\hline MF & $300-3000 \mathrm{kHz}$ & Surface wave, ionospheric wave \\
\hline $\mathrm{HF}$ & $3-30 \mathrm{MHz}$ & $\begin{array}{l}\text { Surface wave, ionospheric wave with significant } \\
\text { refraction, meteor scatter, hop propagation }\end{array}$ \\
\hline VHF & $30-300 \mathrm{MHz}$ & $\begin{array}{l}\text { ionospheric wave (weakly refractive), } \\
\text { Meteor scattering }\end{array}$ \\
\hline UHF & $300-3000 \mathrm{MHz}$ & ionospheric wave (weakly refractive) \\
\hline SHF & $3-30 \mathrm{GHz}$ & ionospheric wave (weakly refractive) \\
\hline EHF & $30-300 \mathrm{GHz}$ & ionospheric wave (weakly refractive) \\
\hline
\end{tabular}

\section{Expansion of the upper atmosphere}

Monitoring of density, winds, temperature, and composition of the neutral atmosphere is an important task, which is also closely related to space weather monitoring.

Space-based systems provide solutions to a large number of practical problems today. Many of these satellites are low-orbital and affected by the neutral atmosphere, which causes their deceleration and premature orbit reduction. This, in turn, shortens the lifetime of a satellite and complicates its tracking. The neutral atmosphere is mainly controlled by solar activity through surface and atmosphere heating by solar radiation and through heat transfer from high-latitude regions, in which ohmic heating is caused by processes and currents in the ionosphere and magnetosphere [Buonsanto, 1999]. Besides, the relationship is being studied between sudden stratospheric warming events and effects of expansion of the upper atmosphere [Liu et al., 2013].

\section{Ionospheric disturbances}

The mechanisms discussed above are related to the direct effect of "severe space weather" on radio devices. The main mechanism of the indirect effect of space 
weather on radio devices are ionospheric disturbances [Buonsanto, 1999; Kutiev et al., 2013]. The ionosphere is a partially ionized gas divided into several basic layers (usually denoted by D, E, and F depending on their distance from Earth's surface). It is located at a height of about 60 to $2000 \mathrm{~km}$ and has a strong influence on radio wave propagation. The interaction of radio waves with the ionosphere depends on frequency, distance from a receiver to a transmitter, ionospheric conditions, and the underlying Earth surface. A fairly detailed description of the radio wave propagation processes can be found in the monographs [Ginzburg, 1960; Budden, 1988].

The main radio devices affected by space weather are HF radio communication devices, surface-to-space communication systems, global navigation systems, over-the-horizon radars, satellite altimeters, and spacebased radars [Goodman, Aarons, 1990]. The stable operation of most of these devices depends on ionospheric conditions [Cannon et al., 2004].

Table 1 lists the main mechanisms of radio wave propagation in different frequency ranges.

In the lower part of the spectrum (VLF, ULF), radio wave propagation can be described as waveguide propaga- tion in the effective waveguide formed by Earth's surface and ionosphere. In the upper part of the spectrum (SHF, $\mathrm{UHF}$ ), radio wave propagation can be considered almost rectilinear, weakly affected by the ionosphere. Between these ranges, the ionospheric impact on radio wave propagation is most considerable, and the HF band is worst affected by solar disturbances and is best suited for designing tools to monitor such effects [Goodman, 2005].

Table 2 shows the main functions of radio equipment, indicating corresponding radio ranges.

The ULF range $(<3 \mathrm{kHz})$ has been studied in sufficient detail [Bannister, 1986; Pappert, Moler, 1978]. In its analysis, the ionosphere and Earth are assumed to be ideal, homogeneous, and with sharp boundaries. The ionosphere acts at these wavelengths as an ideal conductor and generally has no effect on propagation of these waves. Nevertheless, the lower part of the ionosphere, especially the sporadic E layer, can influence radio wave characteristics (mainly phase ones) due to the interference of waves reflected from the regular and sporadic layers [Pappert, 1980].

Some applications of different frequency ranges

\begin{tabular}{|l|l|}
\hline \multicolumn{1}{|c|}{ Range } & \multicolumn{1}{c|}{ Functions } \\
\hline ULF and VLF & Navigation, time and frequency signals \\
\hline LF & navigation, broadcasting \\
\hline MF & amplitude modulation broadcasting \\
\hline HF & $\begin{array}{l}\text { radio communication, standard time signals, radiolocation, } \\
\text { amateur radio communication, positioning systems }\end{array}$ \\
\hline VHF & $\begin{array}{l}\text { Television, broadcasting with signal frequency modulation, } \\
\text { aircraft radio communication }\end{array}$ \\
\hline UHF and EHF & GPS/GLONASS navigation, radiolocation, television \\
\hline
\end{tabular}

Table 2

The basic model for describing oscillation propagation in VLF and LF ranges is waveguide propagation. This approach considers the space between the ionosphere and Earth's surface as a single waveguide. In this case, transverse electric (TE) or transverse magnetic (TM) modes (each with its own characteristics) arise depending on the type of antenna used to generate radiation. However, they are not independent due to the presence of an inhomogeneous magnetic field.

As regards the ionospheric effect on radio wave propagation, the VLF/LF range more strongly depends on ionospheric conditions than ULF, and therefore it is more affected by space weather disturbances. Without considerable space weather disturbances, VLF/LF radio wave propagation is more of less stable. Due to interference of different modes and their propagation conditions, space weather disturbances can cause, for example, sudden phase anomalies (SPA) arising from the enhancement of the D layer during solar flares. When particles penetrate into the polar cap, as during magnetic storms or polar cap absorption events (PCA), propagation conditions also change, resulting in phase and am- plitude signal distortions. Other factors such as ground conductivity have also a strong effect on signal characteristics. These factors are most significant in Polar Regions, which are affected both by the ionosphere and by seasonal dynamics of the underlying surface.

Many researchers combine MF and HF radio ranges because they are characterized by the socalled spatial (sky or ionospheric) wave - a signal path highly refractive in the ionosphere. Moreover, the so-called surface (ground) wave related to radio wave propagation along Earth's surface can be observed in both the ranges. Weakening of the sky wave, for instance, due to absorption in the D layer, increases the probability of observing the ground wave. However, sometimes it is convenient to consider the MF and LF ranges at a time, for instance, to predict characteristics of emission intensity at these wavelengths [Wang, 1985; Ghasemi et al., 2013]. A review of experimental data on MF radio wave propagation can be found in [Knight, 1983; Vilensky et al., 1983].

In the VHF range, space weather effects are largely related to phase and amplitude variations in propagating 
signals due to ionospheric irregularities of different scales. If the irregularities have sufficiently small transverse spatial scales, the phenomenon is observed as scintillation [Basu, Basu, 1981; Basu et al., 1985; Aarons, 1982; Priyadarshi, 2015]; however, if they are relatively large-scale, it appears as smooth changes in characteristics of the received signal.

The main ionospheric effects (signal group delay, Faraday fading, Doppler frequency shift, etc.) depend on the integral value of electron density along a propagation path. This value is usually called total electron content (TEC) and can be measured from data obtained by dual-frequency GPS receivers [Klobuchar, 1975]. Most errors (up to $70 \%$ ) of global satellite positioning can be corrected by taking into account this ionospheric correction. Even greater success can be achieved by knowing the complete three-dimensional distribution of electron density in real time. Ionospheric effects of global large-scale space weather disturbances can be roughly estimated more easily using the global electron content - the total amount of electron plasma in the entire ionosphere [Afraimovich et al., 2008] derived by integrating TEC maps all over the world.

The HF range intermediate between MF and VHF ranges is the most difficult to describe. This is because the critical (plasma) frequencies of the main ionospheric layers (except the D layer) are within this range, and the gyrofrequencies are comparable with the lower boundary of the range. At the same time, HF radio wave propagation can be described in terms of the geometrical optics (hop propagation) [Ginzburg, 1960] and mode propagation [Kurkin et al., 1981], combining features of VHF and LF ranges. High sensitivity to absorption also makes it similar to the lower frequency ranges, especially MF. At the same time, some HF signals with frequencies above the critical frequency can propagate under weak distortion of their paths, but under the strong influence of polarization effects. This makes them close to the VHF range. The existence of irregularities of the order of wavelength, especially at polar latitudes, leads to strong backscattering by natural plasma irregularities (radio aurora) as in the VHF range. The existence of natural ionospheric irregularities smaller than the Fresnel radius results in amplitude-phase fading (scintillation). Doppler frequency shifts exceeding $1 \mathrm{~Hz}$ also make this range similar to VHF and UHF ranges.

Therefore, the space weather impact on the HF range is very strong and includes practically the entire spectrum of effects observable in other ranges: absorption due to ionization during solar flares, absorption in the polar cap, radio aurora, multimode propagation, group and phase delays caused by refraction, Faraday and Cotton-Mouton polarization effects [Ginzburg, 1960; Goodman, 1991], etc.

The extensive use of HF systems requires an understanding of the propagation medium, which in turn is affected by space weather. The strongest space weather disturbance, which affects radio wave propagation, is a geomagnetic storm manifesting itself at all latitudes, including the least disturbed middle latitudes [Akasofu,
1977]. Doppler frequency shifts and signal frequency distortions also depend on the temporal dynamics of the ionospheric channel [Basler et al., 1988] and are responsible for stable receiving. These effects are especially strong at polar and equatorial latitudes.

The main radio equipment that reacts to all or almost all space weather effects is a short-wave over-thehorizon radar with pulsed or continuous signal emission. Their scientific equivalent is SuperDARN pulsed radars [Chisham et al., 2007], ionosonde-direction finders with a continuous chirp signal [Uryadov et al., 2013] or oblique sounding ionosondes [Ivanov et al., 2003]. The principle of the radar operation is to transmit a complex radio signal, which is partially scattered by ionospheric irregularities, partially refracted in the ionosphere, and partially scattered back by Earth's surface. We use data from the Ekaterinburg HF radar (EKB) of ISTP SB RAS to illustrate the space weather impact on radio devices.

\section{OVER-THE-HORIZON RADIOLOCATION AS A METHOD FOR MONITORING SPACE WEATHER EFFECTS}

The main tasks of the over-the-horizon radiolocation are to detect and examine characteristics of scatterers beyond the horizon, using radio wave propagation effects and complex algorithms for rejecting noises from natural sources. The influence of a propagation medium on group and phase delays usually remains considerable. Reviews of these radio devices can be found in [Headrick, Skolnik, 1974; Alebastrov et al., 1984; Headrick, 1990; Principles of Modern Radar, 2010]. In scientific problems, irregularities of a propagation medium (mainly the ionosphere) serve as a scatterer.

As HF radio waves propagate, the emergence of additional ionospheric layers leads to the emergence of additional propagation paths [Tsunoda et al., 2016], and, as a result, complicates matching of the radar range (group delay of a signal) and azimuth to real positions of scattering objects or directions to them [Reinisch et al., 1997; Berngardt et al., 2016; Chen et al., 2016; Warrington et al., 2016]. The frequency dependence of propagation paths causes strong phase distortions, thus making the detection of complex signals over long paths even more difficult. Moreover, the signal amplitude can vary due to defocusing/focusing of the signal [Berngardt et al., 2016] and its absorption in the lower ionospheric layers [Berngardt et al., 2016; Gauld et al., 2002; Settimi et al., 2014; Settimi et al., 2015; Sonnenschein et al., 1997]. All these are supplemented with the previously described effects: changes in group and phase delays and polarization distortions. Even accurate measurements of the speed of scatterers require us to correctly take into account the background ionosphere [Gillies et al., 2011].

The operation of over-the-horizon radio devices is maintained with systems for modeling radio signal propagation in an inhomogeneous magnetized ionospheric plasma [Fridman et al., 2016; Landeau et al., 
1997; Reinisch et al., 1997; Settimi et al., 2015; Warrington et al., 2016] because they can reduce ionospheric errors [Reinisch et al., 1997; Berngardt et al., 2015b]. To correctly solve the problem of signal propagation, we should know the propagation medium or at least have a good model of the medium. There are various models for predicting and correcting propagation characteristics in applications to diverse radio systems (for example, IONCAP, VOACAP, ICEPAC, ASAPS), designed mainly to predict different ionospheric characteristics [Zolesi, Cander, 2014]. Median monthly ionospheric models, developed more than 50 years ago and constantly improved [Bilitza et al., 2014, 2017], can also be employed to predict the operation of $\mathrm{HF}$ radio devices under different conditions. Examples of such improvements are NeQuick [Radicella, Leitinger, 2001] and PIM [Daniell et al., 1995]. One of the common models is the International Reference Ionosphere (IRI) adjusted by different data [Bilitza et al., 2014, 2017; Settimi et al., 2015]. Most of these models are either statistical or smoothed, thus impeding effective monitoring of local changes, which are most pronounced at high latitudes.

The most accurate is the real-time monitoring of ionospheric characteristics based on data from networks of instruments and their use to correct radio propagation models [Settimi et al., 2015; Bilitza et al., 2017; Hernández-Pajares et al., 2017].

The EKB radar $\left(56.5^{\circ} \mathrm{N}, 58.5^{\circ} \mathrm{E}\right)$, put into trial operation in December 2012, is a pulsed decameter coherent radar installed jointly with the Institute of Geophysics of the Ural Branch of the Russian Academy of Sciences (IGP UrB RAS) at the IGP UrB RAS observatory Arti. The transmitter/receiver equipment of the radar was developed at the University of Leicester (Great Britain) and purchased at the expense of SB RAS. The installation of the radar array was funded by Rosgidromet (Russian Hydrometeorological Service). At present, this radar is the only scientific pulse decameter over-the-horizon radar in the Russian Federation.

The transceiver antenna system of the radar is a linear phased array; it provides a $3-6^{\circ}$ beamwidth depending on frequency and a $50^{\circ}$ field of view scanned by fixed beam positions one by one. The spatial and temporal resolutions of the radar are $15-45 \mathrm{~km}$ and $2 \mathrm{~min}$ respectively. The $8-20 \mathrm{MHz}$ frequency range enables the radar to operate as an over-the-horizon radar, and the peak power of $10 \mathrm{~kW}$ ensures its operation in the range up to $3500-4500 \mathrm{~km}$. Short sounding signals provide a low (about $600 \mathrm{~W}$ ) mean radar power, thus enabling it to operate in the round-the-clock monitoring regime. An approximate field of view of the radar is shown in Figure 1. Refractive effects make this sector somewhat larger; and when solving specific problems it is necessary to take into account conditions of the background ionosphere to calculate the region from which a signal comes.

In the geomagnetic storm main phase, the ionospheric

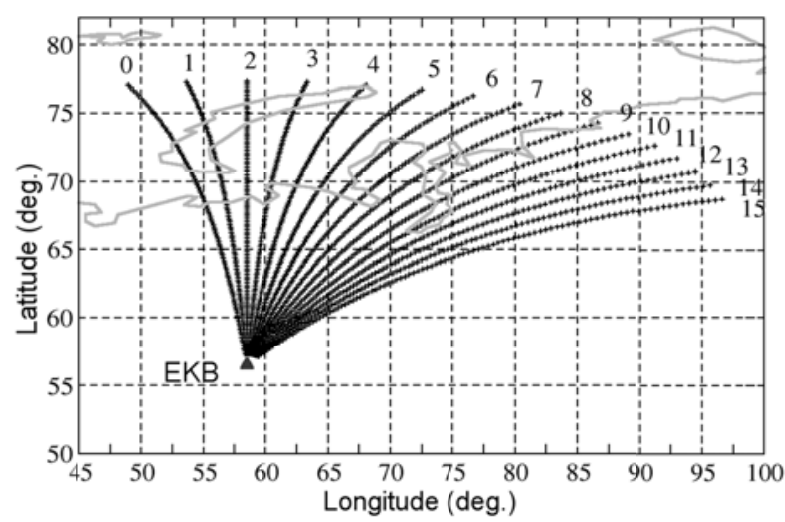

Figure 1. Approximate field of view of the EKB radar in geographical coordinates, excluding refraction in the ionosphere. Numbers indicate radar beam numbers

plasma frequency can decrease to $50 \%$ of its pre-storm value and then recover within a few days. The refraction coefficient, radio signal propagation paths, group and phase propagation delays also vary a great deal.

Electron density variations in the $\mathrm{E}$ and $\mathrm{F}$ layers usually cause a radio signal propagation path to distort; the lower is the frequency, the stronger are these distortions.

The weakest distortions that amount to a change of group delays in ionospheric propagation or to polarization distortions associated with Faraday fading [Ginzburg, 1960; Budden, 1988] occur in VHF and UHF ranges. In this case, the main effect refers to the errors in determining the range calculated from group or phase delays. Such errors are peculiar to various radar systems and are widely known in data of Global Positioning Systems (GPS) [Klobuchar et al., 1987]. Faraday fadings can lead to additional variations in signal power, which are noticeable in radio astronomical observations [Afraimovich, 2007].

The main factor affecting HF radio wave propagation is refraction caused by a large-scale ionospheric irregularity. To illustrate the effect of variations in the background ionospheric characteristics on a radio signal (Figure 2), we show the power of a scattered signal as a function of the radar range and time as inferred from the EKB radar data for three selected days: May 19, 2016, August 30, 2016, and September 22, 2016. Figure 2, a$c$ shows that to the nighttime (22-24, 00-08 LST) correspond large radar ranges (zone II); and to the daytime (09-19 LST, zone I), small radar ranges. Figure $2, b$ indicates that diurnal variations of the radar range can exceed $1000 \mathrm{~km}$. Figure 2, $d$ explains this effect in terms of daytime (black color) and nighttime (gray color) refraction. It is seen that with a nighttime decrease in the electron density $N_{\mathrm{e}}$ (right in Figure 2,d) and an increase in its maximum, the trajectory becomes longer, and so does the radar (group) delay to the boundary of the dead zone. This effect is a regular daily one and depends on the three-dimensional electron density distribution over the entire propagation path of the signal. 


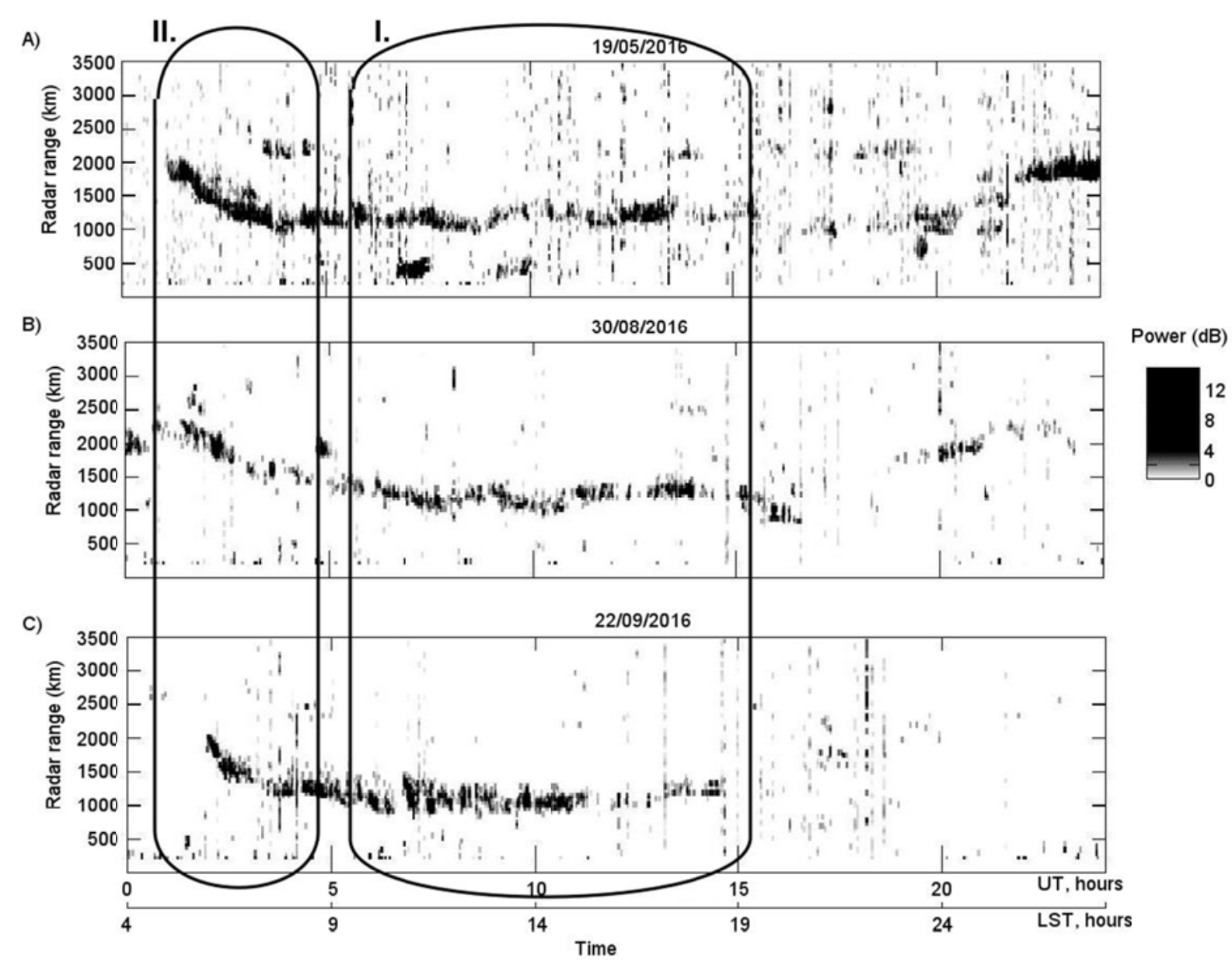

D)

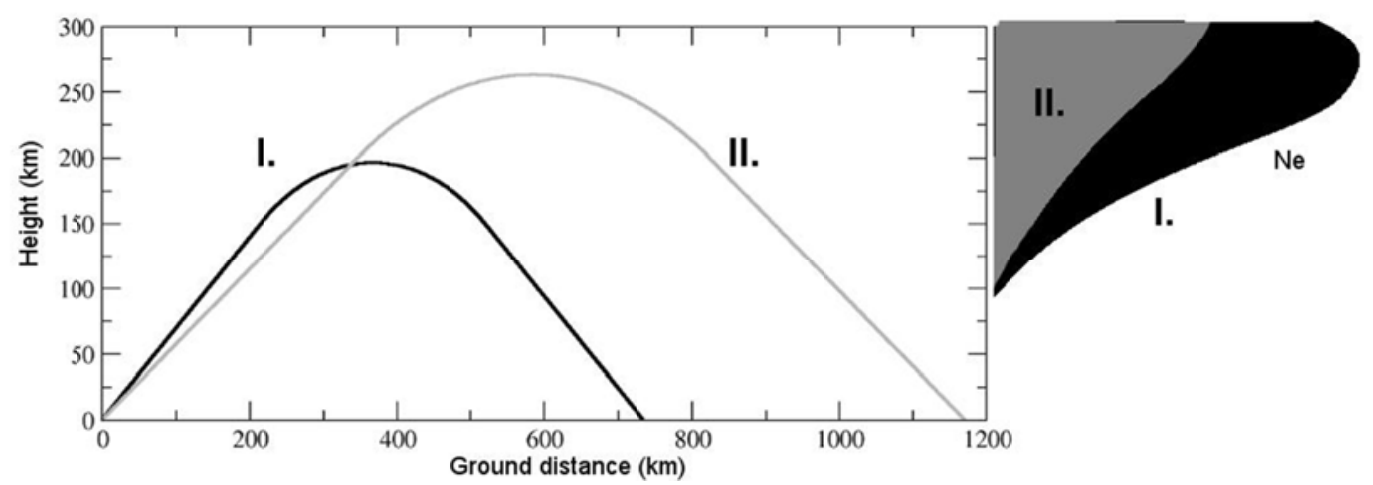

Figure 2. Power of a scattered EKB radar signal for May 19, 2016 (a), August 30, 2016 (b), and September 22, 2016 (c) vs radar range of scattering and time, $\mathrm{LST}=\mathrm{UT}+4$; an approximate signal propagation trajectory in daytime (black) and nighttime (gray) $(d)$

Eigenwaves propagating in the magnetized ionosphere are ordinary and extraordinary waves with different polarizations, depending on the angle with the magnetic field. When waves propagate in the ionosphere at different velocities, their superposition undergoes Faraday fadings. The fading phase in the first approximation is proportional to the total electron content [Kravtsov, Orlov, 1980].

The ionosphere being a dispersive medium, differ- ent frequencies propagate in it with different velocities. Hence, propagating in the medium, a signal is distorted. Distortions of narrowband HF signals were discussed, for example, in [Zasenko et al., 1993]. Such distortions can be related both to the refraction and signal focusing effects and to the frequency dispersion of the ionospheric absorption. Besides F-layer ionization, electron density can change in the $\mathrm{D}$ and $\mathrm{E}$ layers, thus causing higher radio wave absorption. 
Radio wave absorption occurs when the effective frequency of collision of electrons with other particles becomes sufficiently high. Thus the wave energy is transferred to heavier particles - ions and neutrals, converting into heating. This occurs most often during solar flares when the electron density in the D layer increases [Rogov et al., 2015]. Basically, the absorption is high in the HF range and at lower frequencies, while at VLF frequencies it becomes rather low.

Figure 3,a-f shows the behavior of the scattered signal power during several X-ray flares: May 08, 2014 (M5.2), April 11, 2013 (M6.5), October 25, 2013 (M9.4), and respective variations in X-rays as derived from GOES data. It is seen that often during X-ray flares the signal power can decrease substantially without changing the scattering range; hence, the main effect is associated with the appearance of an ionized region that has weak refraction but high absorption, such as the D layer. Figure $3, g$ illustrates a change in signal intensity during propagation in the presence of a strongly ionized lower region; Figure $3, h$, in its absence. The dashed line in Figure 3, $g$ schematically shows the signal amplitude that decreases as the signal passes the absorbing layer. The signal trajectory changes slightly, but the signal amplitude drops considerably as the signal propagates in the absorbing layer.

Ionization of lower ionospheric layers, which is caused by energetic solar protons and leads to radio wave absorption in the polar cap (PCA), usually lasts from hours to several days and is particularly critical during HF radio wave propagation along polar radio paths [Perrone et al., 2004], resulting in a large decrease in the
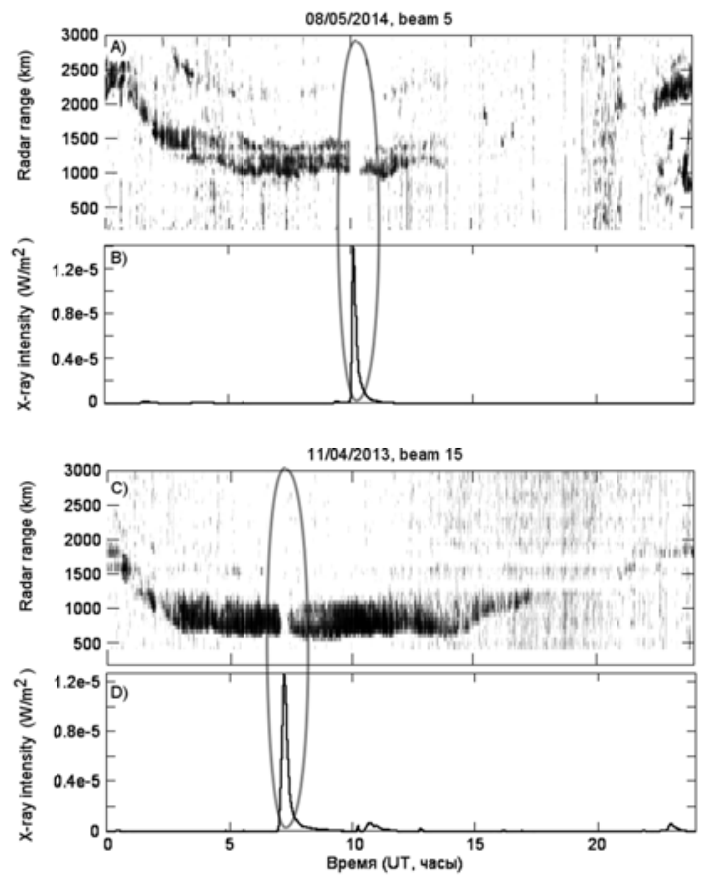

amplitude of the propagating wave (up to $100 \mathrm{~dB}$ ).

The existence of abrupt changes in background parameters of the ionosphere or solar wind, as well as steep spatial gradients of ionospheric parameters, brings about the formation of various irregularities. In the presence of eigenoscillations and variations in the magnetosphere-ionosphere-atmosphere system under the action of driving forces, these effects can oscillate in space and time according to complex laws determined by characteristics of the system's eigenoscillations and the dynamics of external action. In practice, this leads to the occurrence of spatio-temporal variations of all these parameters with different space-time scales: planetary waves [Liu et al., 2010], internal gravity waves [Hunsucker, 1982], etc., and, thus, to the temporal modulation of the above effects.

Figure 4 shows the power of a scattered EKB radar signal as a function of the radar range of scattering and time in the presence of traveling ionospheric disturbances. Figure 4, $a, b$ illustrates cases of large-scale irregularities of the $\mathrm{F}$ layer without formation of an additional signal propagation mode (path), which generally reduce to a change in the shape of the electron density profile without changing its monotony. Similar irregularities are usually considered as internal or acoustic-gravity waves [Oinats et al., 2016]. The principle of formation of such effects is similar to that shown in Figure $2 d$. Figure 4, $c, d$ demonstrates cases of largescale irregularities with the formation of an additional propagation mode, which usually reduce to the formation of wavelike vertical disturbances disrupting the mono-
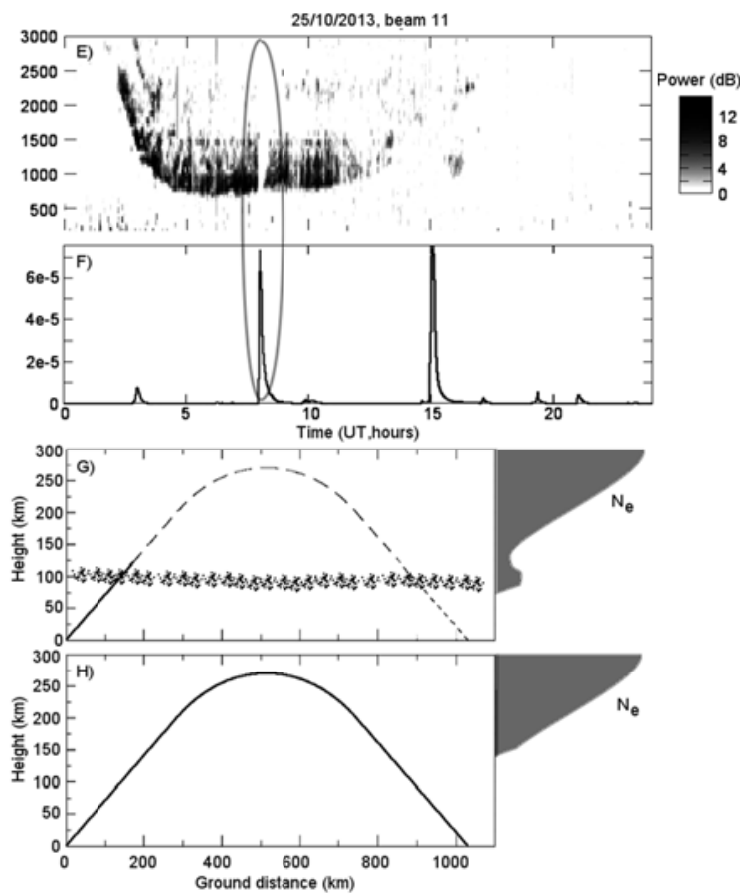

Figure 3. Power of a scattered EKB radar signal vs the radar range of scattering and time during X-ray flares M5.2 on May 08, 2014 (a), M6.5 on April 11, 2013 (c), M9.4 on October 25, 2013 (e), and respective variations in X-rays as inferred from GOES data $(b, d, f)$; approximate trajectories and amplitudes of signals as they propagate in the presence $(g)$ and in the absence $(h)$ of the absorbing layer at a height of $100 \mathrm{~km}$ 
tonic electron density profile and appearing as filaments in the range-time diagram, the direction of these filaments indicating the direction of motion of these irregularities [Stocker et al., 2000]. Similar irregularities arose, for example, in the passage of waves from the Chelyabinsk bolide on February 15, 2013 [Berngardt et al., 2015c; Kutelev, Berngardt, 2013] or in the passage of shock waves from earthquakes [Ogawa et al., 2012; Berngardt et al., 2017].

Wave phase changing due to propagation in the refracting ionosphere, the signal acquires a time-varying phase shift, described in the first approximation by the Doppler frequency shift. The frequency shift in the HF range can be up to tens of hertz depending on external conditions.

As the spatial scale of the irregularities decreases, the oscillations become faster and more pronounced in the Doppler frequency shift. Figure 5 shows the power and Doppler frequency shift of the scattered EKB radar signal in velocity units (hereinafter called Doppler velocity) as a function of the radar range of scattering and time during such medium-scale irregularities.

A close correspondence can be seen between the Doppler velocity and the power variations associated probably not only with the motion of the reflection point along the range (Figure 2,d), but also with the focusing effects [Stocker et al., 2000; Kutelev, Berngardt, 2013], i.e., they are a combination of the effects discussed in Figure 4. It is clear to what a further reduction in scales of irregularities will lead - to signal fadings with even smaller periods, i.e., to the scintillation effect.

Ionospheric irregularities generating signal scintillation have been studied in many works, in particular their dependence on various manifestations of solar and geomagnetic activity. A positive correlation between their appearance and solar activity index is typical for equatorial and high latitudes [Aarons et al., 1980; Rino, Matthews, 1980]. The scintillation can be caused by irregularities of different scales - from meter to kilometer, including medium-scale irregularities, whose dimensions are comparable and less than the radius of the Fresnel zone [Basu, Basu, 1981; Basu et al., 1985, 1988; Mullen et al., 1985; Aarons, 1982; Weber et al., 1985; Groves et al., 1997; Wernik et al., 2003; Gherm et al., 2011]. Notice that the scintillation is more intense at equatorial latitudes, although they can also occur at high latitudes, and is largely associated with the development

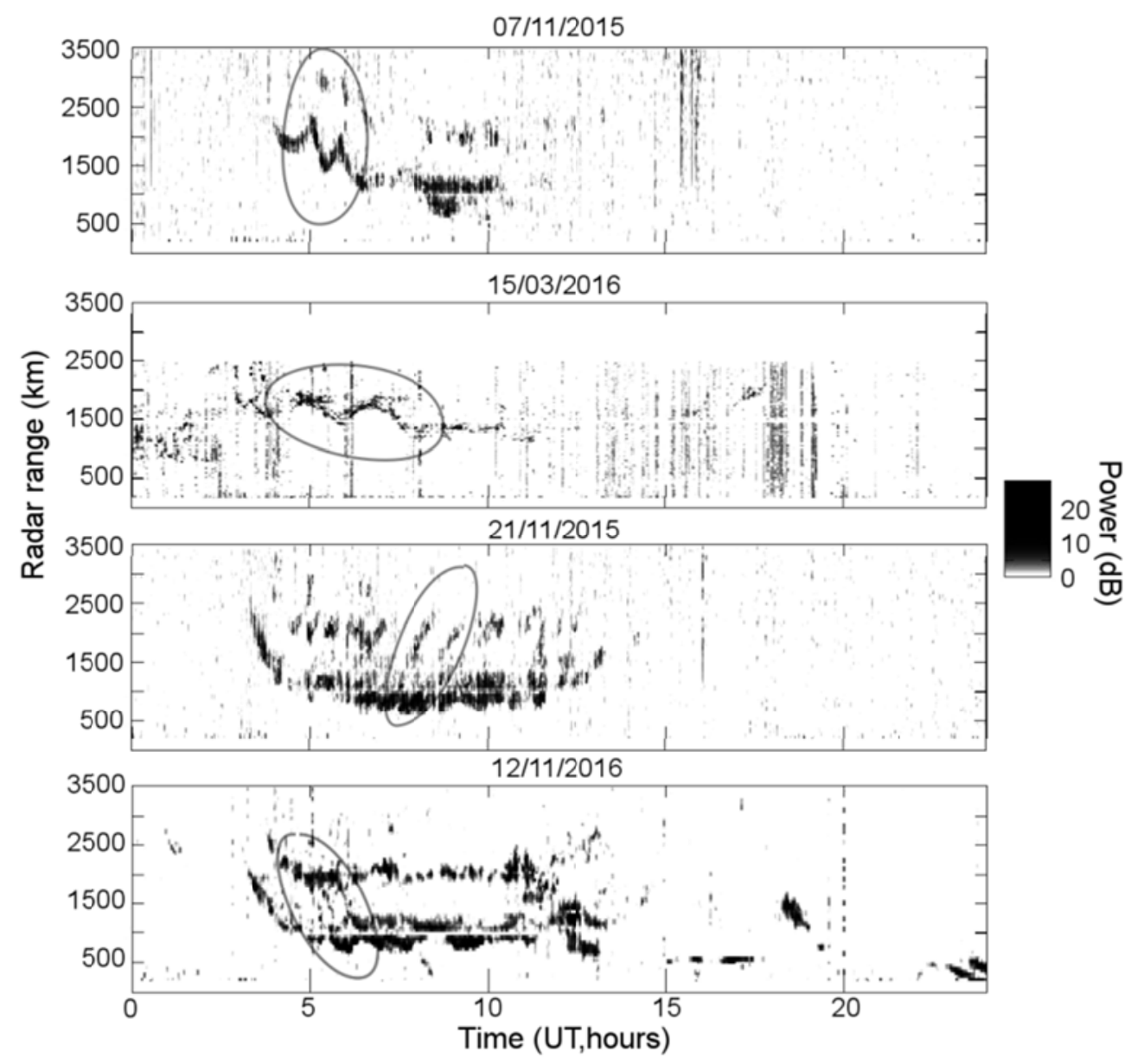

Figure 4. Power of a scattered EKB radar signal vs the radar range of scattering and time in the presence of traveling ionospheric disturbance - large-scale irregularities of the F layer - without $(a, b)$ and with formation $(c, d)$ of an additional propagation mode 
of various instabilities [Tsunoda, 1988]. In the equatorial ionosphere, the main mechanism of scintillation is the plume (plasma irregularity). Plumes have characteristic dimensions of the order of several hundreds of kilometers in horizontal and vertical directions. At high latitudes, scintillation is associated with the growth of small-scale irregularities [Rino, Matthews, 1980; Aarons, 1982]. Scintillation at middle latitudes is usually caused by a combination of high-latitude mechanisms and the effect of irregularities in the subauroral ionosphere, including the emergence of sporadic layers [Goodman, 1967].

Small-scale irregularities (with a characteristic scale of the order of half the signal wavelength) can also lead to substantial backscattering in the HF and VHF ranges, known as radio aurora. There are numerous types of such irregularities typical for both polar [Bagaryatsky, 1961; Sverdlov, 1982; Haldoupis, 1989] and equatorial ionospheric regions [Patra et al., 2014; Chau, Kudeki, 2013]. A feature of most of these irregularities is their extension along geomagnetic field lines.

The morphology of the high-latitude radio aurora has been extensively studied. Its main characteristic is the relationship with the polar oval position (which, for example, is responsible for the well-known diurnal dependence of the radio aurora with the nighttime enhancement) and electric field intensity, which is one of the main effects accompanying space weather disturbances. Radio aurora has been the objective of many studies [Bagaryatsky, 1961; Sverdlov, 1982; Uryadov et al., 2013], including recent research based on EKB radar data [Berngardt et al., 2015a]. Radio aurora observations by the SuperDARN radar network are reviewed in [Chisham et al., 2007].

It should be noted that the probability of the occurrence of small-scale irregularities is often associated with regions of intensive currents. As already mentioned, a change in solar wind characteristics leads to a change in this current system, in particular to the expansion of the polar oval region in both equatorial and polar directions.

The ionospheric currents accompanying the polar oval move to more equatorial regions and manifest themselves in data from magnetic stations due to generation of magnetic fields [Rostoker, 1972], thus allowing us to estimate the degree of geomagnetic disturbance in the ionosphere. Moreover, indices that feature the degree of growth of the polar oval can be constructed
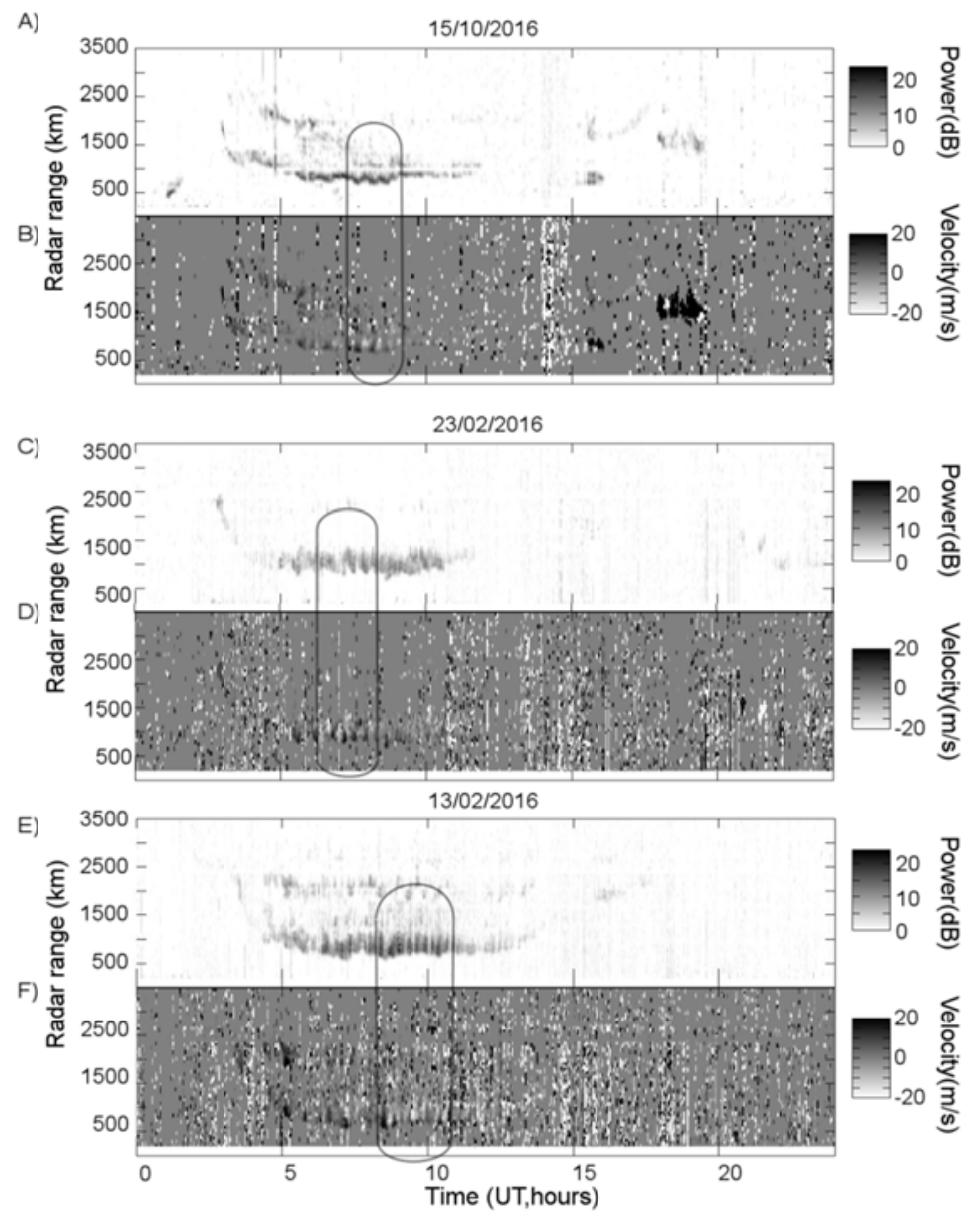

Figure 5. Power of a scattered EKB radar signal $(a, c, e)$ and Doppler frequency shift in velocity units $(b, d, f)$ vs of the radar range of scattering and time in the presence of medium-scale irregularities 


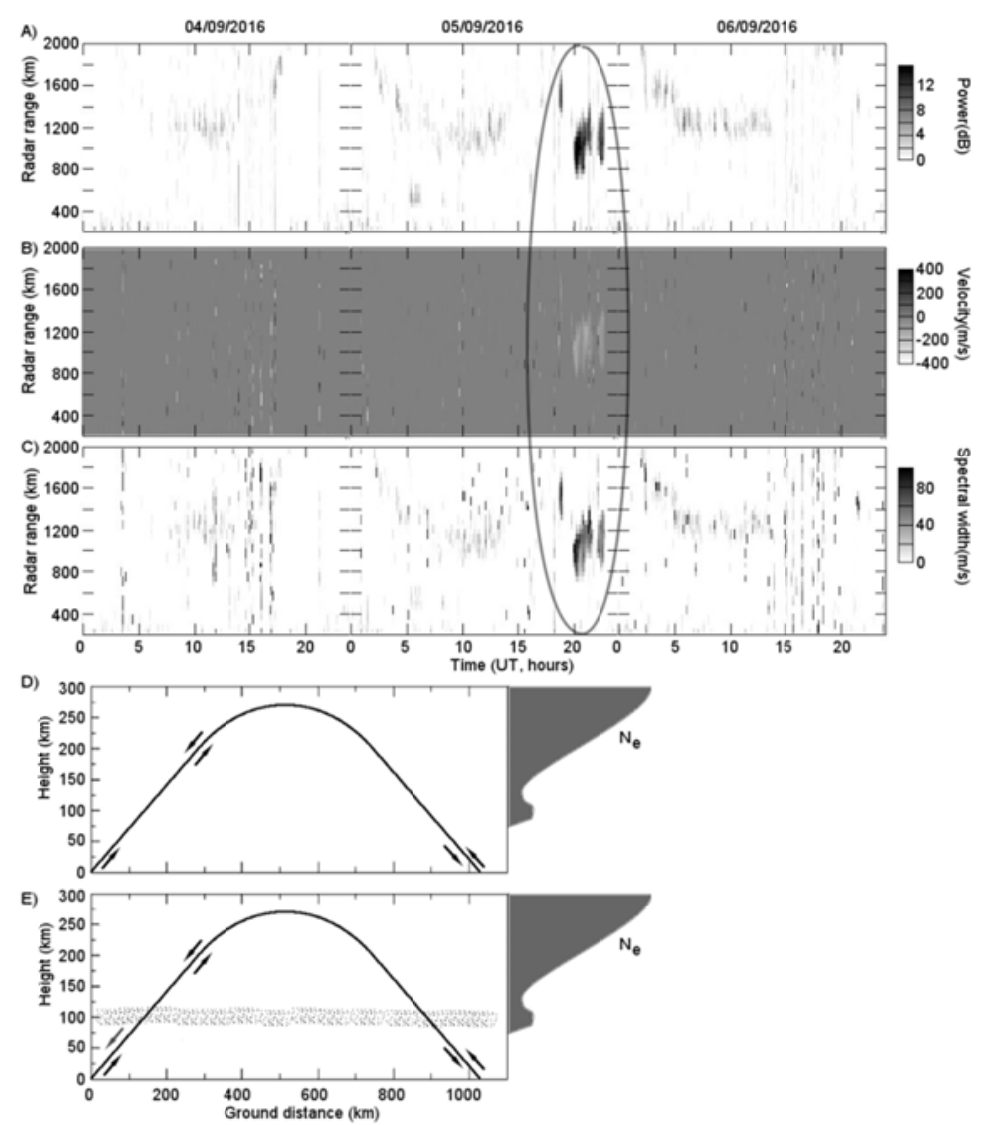

Figure 6. Power of a scattered EKB radar signal (a), the Doppler velocity $(b)$, and the signal spectral width in velocity units (c) vs the radar range of scattering and time; approximate paths and amplitudes of signals propagating without $(d)$ and with $(f)$ a layer with irregularities at a height of $100 \mathrm{~km}$

through a direct analysis of interplanetary magnetic field disturbances (Troshichev et al., 2006).

Figure 6 shows the power of a scattered EKB radar signal (a), the Doppler velocity $(b)$, and the spectral width in units of the Doppler velocity $(c)$ as a function of the radar range of scattering and time over the period from September 04 to 06, 2016 along beam 7 .

The Figure depicts a large spatial region occupied by the radio aurora, and high equivalent Doppler velocities of irregularities exceeding $200 \mathrm{~m} / \mathrm{s}$. A signal scattered by such irregularities is very complex and has a significant temporal variability, which is confirmed by the large spectral width.

Notice that both electron density and electric field variations can cause changes in the Doppler frequency shift of a received signal. In this case, the Doppler frequency shift of a signal scattered by magnetically oriented irregularities is modulated [Bland et al., 2014]. These effects, observed with the EKB radar, have been analyzed in [Mager et al., 2015; Chelpanov et al., 2016].

Examples of such oscillations are shown in Figure 7. The Figure shows the power of a scattered signal ( $a, b$, $d)$ and the Doppler velocity $(b, d, f)$. This Figure indicates that there are radio-aurora regions where the Doppler frequency shift reverses sign (marked with an oval). Such quasi-periodic sign reversals can be either longperiod (Figure 7, $a, b$ ) or short-period (Figure 7, $c-f$ ).
Given that plasma at large heights can be considered magnetized, the Doppler velocity of irregularities is determined by the $\mathbf{E} \times \mathbf{B}$ drift, and Doppler velocity oscillations can be explained by the electric field rotation both due to a change in the structure of currents in the E layer [Chisham et al., 2007] and due to propagation of waves of various types through the magnetosphere [Chelpanov et al., 2016].

\section{CONCLUSION}

This paper is an attempt to review effects of space weather disturbances on operation of radio devices. The emphasis is on space weather impact on propagation of HF radio waves. The ISTP SB RAS EKB radar data are used to demonstrate some manifestations of this impact. Examples are given of changes in the group delay of a signal scattered by Earth's surface, which are associated with the dynamics of the background electron density. Absorption of such a scattered signal during solar flares is also exemplified. The effects of large-scale irregularities on the group delay and structure of a signal scattered from Earth's surface, as well as medium-scale irregularities on the group delay, and the Doppler shift of the scattered signal frequency are demonstrated. Examples are given of the appearance of a signal, scattered by magneto-oriented irregularities, and variations in the Doppler frequency shift, which are most often associated with 


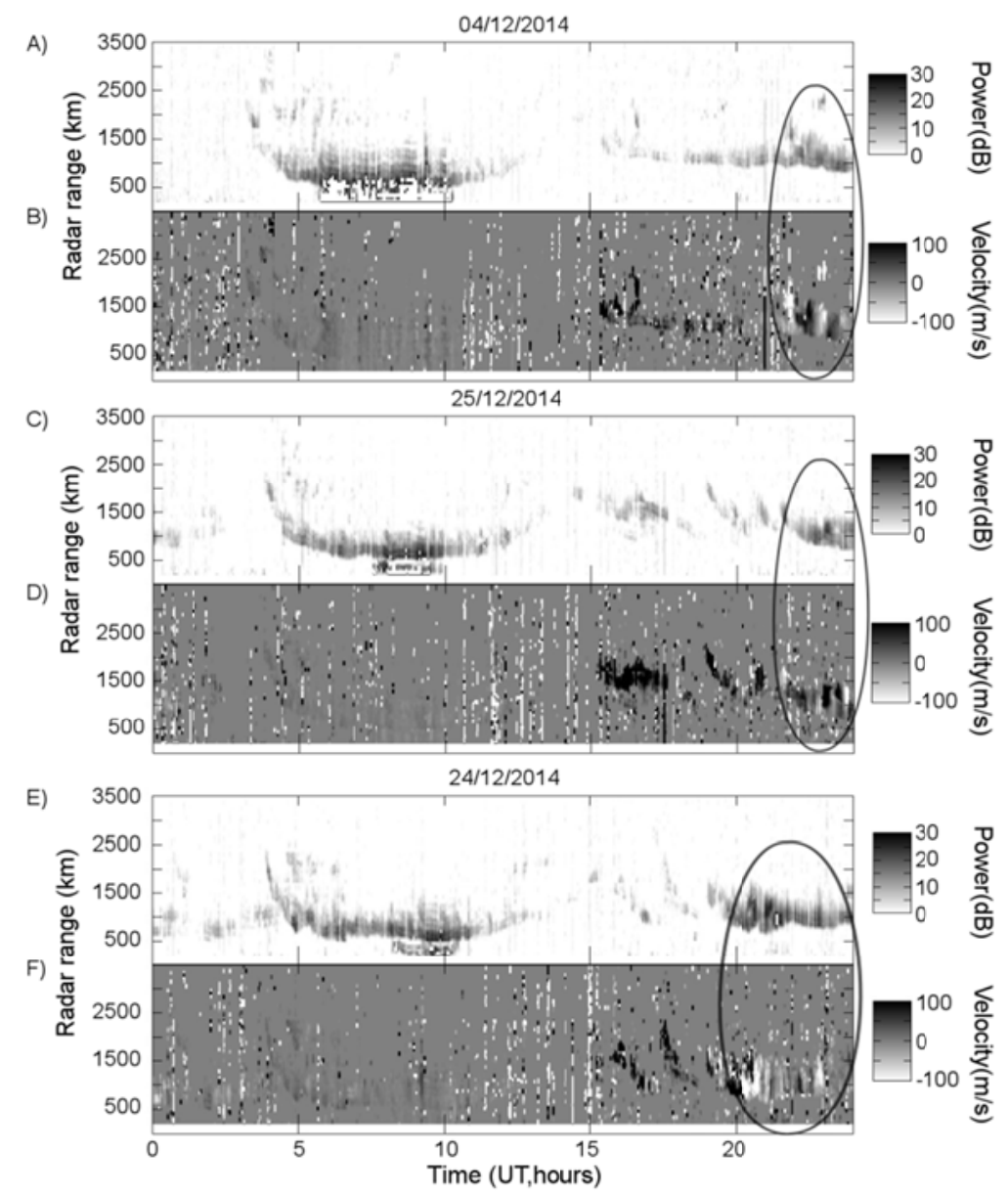

Figure 7. Power of a scattered signal ( $a, c, e)$ and Doppler velocity $(b, d, f)$ during the observation of ULF oscillations of radioaurora characteristics on December 04, 2014, December 25, 2014, and December 24, 2014. Ovals indicate regions of oscillation observations

magnetospheric waves. Thus, it has been shown that pulsed decameter radars, including SuperDARN radars, are multifunction sensitive devices which are affected by various space weather factors and allow monitoring of space weather effects in the ionosphere.

The author is grateful to NOAA for providing GOES data [http://www.swpc.noaa.gov/products/x-ray-flux], to A.V. Tashchilin, S.B. Lunyushkin, and M.V. Uspensky for useful and fruitful discussions. Experimental data were obtained with the EKB radar of ISTP SB RAS under the project II.12.2.3. The work was funded by RAS Presidium Program 7.

\section{REFERENCES}

Aarons J. Global morphology of ionospheric scintillations. Proc. IEEE. 1982, vol. 70, no. 4, pp. 360-378. DOI: 10.1109/ PROC.1982.12314.

Aarons J., MacKenzie E., Bhavnani K. High latitude analytic formulas for scintillation levels. Radio Sci. 1980, vol. 15, no. 1, pp. 115-127. DOI: 10.1029/RS015i001p00115.

Afraimovich E.L. Ionospheric Faraday modulation of radioastronomical signals intensity. Doklady Akademii nauk [Doklady Earth Sci]. 2007, vol. 417, no. 6, pp. 818-822. (In Russian).
Afraimovich E.L., Astaf'eva E.I., Berngardt O.I., et al. Mid-latitude amplitude scintillations of GPS signals and GPS failures at the auroral oval boundary. Radiophysics and Quantum Electronics. 2004, vol. 47, no. 7, pp. 453-468. DOI: 10.1023/B:RAQE.0000047237.67771.bc.

Afraimovich E.L., Zherebtsov G.A., Smol'kov G.Ya. Total failure of the satellite navigation system GPS during December 6, 2006 solar flare. Doklady Akademii nauk [Doklady Earth Sci.]. 2007, vol. 416, no. 6, pp. 817-821. (In Russian).

Afraimovich E.L., Astafyeva E.I., Oinats A.V., et al. Global electron content: a new conception to track solar activity. Ann. Geophys. 2008, vol. 26, no. 2, pp. 335-344. 617 p. DOI: 10.5194/ angeo-26335-2008.

Akasofu S.-I. Physics of Magnetospheric Substorms. Boston, D. Reidel Publishing Co., 1977, DOI: 10.1007/978-94010-1164-8.

Alebastrov V., Gojhman Je., Zamorin I. Osnovy zagorizontnoi radiolokatsii [Fundamentals of Over-the-Horizon Radiolocation]. Moscow, Radio i Svyaz 'Publ., 1984, 256 p. (In Russian).

Al'pert Ya.L. The current state of the problem of ionospheric studies. III. Some additional questions. Uspekhi fizicheskikh nauk [Soviet Physics-Uspekhi (Adv. Phys. Sci.)]. 1949, vol. 38, iss. 3, pp. 309-337. DOI: 10.3367/UFNr.0038.194907a.0309. 
Aurorasourus. 2016. URL: http://aurorasaurus.org (accessed December 12, 2016).

Bagaryatsky B.A. Radar reflections from aurorae. Uspekhi fizicheskikh nauk [Soviet Physics-Uspekhi (Adv. Phys. Sci.)]. 1961, vol. 73, no. 2, pp. 197-241. DOI: 10.3367/UFNr.0073. 196102a. 0197. (In Russian).

Baker D.N., Jaynes A.N., Hoxie V.C., et al. An impenetrable barrier to ultrarelativistic electrons in the Van Allen radiation belts. Nature. 2014, vol. 515, no. 7528, pp. 531-534. DOI: 10.1038 /nature13956.

Bala B., Lanzerotti L.J., Gary D.E., Thomson D.J. Noise in wireless systems produced by solar radio bursts. Radio Sci. 2002, vol. 37, no. 2, pp. 2-1-2-7. DOI: 10.1029/2001RS002481.

Bannister R. Simplified formulas for ELF propagation at shorter distance. Radio Sci. 1986, vol. 21, no. 3, pp. 529-537. DOI: 10.1029/RS021i003p00529.

Barlow W.H. On the spontaneous electric currents observed in the wires of the electric telegraph. Phil. Trans. R. Soc. 1849, vol. 139, pp. 61-72.

Barnard L., Scott C., Owens M., et al. The Solar Stormwatch CME catalogue: Results from the first space weather citizen science project. Space Weather. 2014, vol. 12, no. 12, pp. 657-674. DOI: 10.1002/ 2014SW001119.

Basler R.P., Price G.H., Tsunoda R.T., Wong T.L. Ionospheric distortion of HF signals. Radio Sci. 1988, vol. 23, no. 4, pp. 569-579. DOI: 10.1029/RS023i 004p00569.

Bastian T.S., Benz A.O., Gary D.E. Radio emission from solar flares. Annual Review of Astronomy and Astrophysics. 1998, vol. 36, no. 1, pp. 131-188. DOI: 10.1146/annurev. astro.36.1.131.

Basu S., Basu Su. Equatorial scintillations - a review. J. Atmos. Solar-Terr. Phys. 1981, vol. 43, no. 5-6, pp. $473-$ 489. DOI: 10.1016/0021-9169(81)90110-0.

Basu Su., Basu S., MacKenzie E., Whitney H.E. Morphology of phase and intensity of scintillations in the auroral oval and polar cap. Radio Sci. 1985, vol. 20, no. 3, pp. 347-356. DOI: 10.1029/RS020i003p00347.

Basu Su., Basu S., Weber E.J., Coley W.R. Case study of polar cap scintillation modeling using DE 2 irregularity measurements at $800 \mathrm{~km}$. Radio Sci. 1988, vol. 23, no. 4, pp. 545-553. DOI: 10.1029/RS023i004 p00545.

Béland J., Small K. Space Weather Effects on Power Transmission Systems: The Cases of Hydro-Qu'ebec and Transpower New Zealand Ltd. Effects of Space Weather on Technology Infrastructure. Netherlands, Kluwer Academic Publishers, 2004, pp. 287-299. DOI: 10.1007/1-4020-2754-0_15.

Benz A.O. Millisecond radio spikes. Solar Phys. 1986, vol. 104, pp. 99-110. DOI: 10.1007/BF00159950.

Berngardt O.I., Kutelev K.A., Potekhin A.P. SuperDARN scalar radar equations. Radio Sci. 2016, vol. 51, no. 10, pp. 1703 1724. DOI: $10.1002 / 2016$ rs006081.

Berngardt O.I., Zolotukhina N.A., Oinats A.V. Observations of field-aligned ionospheric irregularities during quiet and disturbed conditions with EKB radar: First results. Earth, Planets and Space. 2015a, vol. 67, 143. DOI: 10.1186/s40623015-0302-3.

Berngardt O.I., Kutelev K.A., Kurkin V.I., et al. Bistatic sounding of high-latitude ionospheric irregularities using a decameter EKB radar and an UTR-2 radio telescope: First results. Radiophysics and Quantum Electronics. 2015b. vol. 58, no. 6, pp. 390-408. DOI: 10.1007/s11141015-9614-1.

Berngardt O.I., Perevalova N.P., Dobrynina A.A., et al. Toward the azimuthal characteristics of ionospheric and seismic effects of Chelyabinsk meteorite fall according to the data from coherent radar, GPS, and seismic networks. J. Geophys. Res.: Space Phys. 2015c, vol. 120, no.12, pp. 10754-10771. DOI: 10.1002/2015JA021549.

Berhgardt O.I., Perevalova N.P., Podlesnyi A.V., Kurkin V.I., Zherebtsov G.A. Vertical midscale ionospheric disturbances caused by surface seismic waves based on Irkutsk chirp ionosonde data in 2011-2016. J. Geophys. Res.: Space Phys. 2017, vol. 122, pp. 4736-4754. DOI: 10.1002/2016JA023511.

Bilitza D., Altadill D., Zhang Y., et al. The International Reference Ionosphere 2012 - a model of international collaboration. J. Space Weather and Space Climate. 2014, vol. 4, A07. 12 p. DOI: 10.1051/swsc/ 2014004.

Bilitza D., Altadill D., Truhlik V., Shubin V., Galkin I.A., Reinisch B., and Huang X. International Reference Ionosphere 2016: from ionospheric climate to real-time weather predictions. Space Weather. 2017, vol. 15, iss. 2, pp. 418-429. DOI: 10.1002/2016sw001593.

Bland E.C., McDonald A.J., Menk F.W., Devlin J.C. Multipoint visualization of ULF oscillations using the Super Dual Auroral Radar Network. Geophys. Res. Lett. 2014, vol. 41, no. 18, pp. 6314-6320. DOI: 10.1002/2014 g1061371.

Boteler D.H. Geomagnetically induced currents: present knowledge and future research. IEEE Transactions on Power Delivery. 1994, vol. 9, no. 1, pp. 50-58. DOI: 10.1109/61.277679.

Budden K.G. The Propagation of Radio Waves: The Theory of Radio Waves of the Low Power in the Ionosphere and Magnetosphere . Cambridge University Press, 1988, 688 p.

Buonsanto M.J. Ionospheric storms - a review. Space Sci. Rev. 1999, vol. 88, no. 3, pp. 563-601. DOI: 10.1023/A: 1005107532631.

Campbell W.H. Induction of auroral zone electric currents within the Alaska pipeline. Pure and Applied Geophysics. 1978, vol. 116, no. 6, pp. 1143-1173. DOI: 10.1007/BF00874677.

Cannon P.S., Angling M.J., Heaton J.A.T., et al. The Effects of Space Weather on Radio Systems with a focus on HF systems. Effects of Space Weather on Technology Infrastructure. Netherlands, Kluwer Academic Publishers, 2004, pp. 185201. DOI: $10.1007 /$ 1-4020-2754-0_10.

Chau J.L., Kudeki E. Discovery of two distinct types of equatorial $150 \mathrm{~km}$ radar echoes. Geophys. Res. Lett. 2013, vol. 40, no. 17, pp. 4509-4514. DOI: 10.1002/grl.50893.

Chelpanov M.A., Mager P.N., Klimushkin D.Yu., et al. Experimental evidence of drift compressional waves in the magnetosphere: an Ekaterinburg coherent decameter radar case study. J. Geophys. Res.: Space Phys. 2016, vol. 121, no. 2, pp. 13151326. DOI: 10.1002/2015 JA022155.

Chen X.C., Lorentzen D.A., Moen J.I., et al. F-region ionosphere effects on the mapping accuracy of SuperDARN HF radar echoes. Radio Sci. 2016, vol. 51, no. 5, pp. 491-506. DOI: 10.1002/2016rs005957.

Chernov G.P. Fine Structure of Solar Radio Bursts. Springer, 2011, 300 p. DOI: 10.1007/ 978-3-642-20015-1. 
(Astrophys. Space Sci. Library. vol. 375).

Chisham G., Lester M., Milan S.E., et al. A decade of the Super Dual Auroral Radar Network (SuperDARN): scientiffic achievements, new techniques and future directions. Surv. Geophys. 2007, vol. 28, no. 1, pp. 33-109. DOI: 10.1007/s10712-007-9017-8.

Daniell R.E., Brown L.D., Anderson D.N., et al. Parameterized ionospheric model: a global ionospheric parameterization based on first principles models. Radio Sci. 1995, vol. 30, no. 5, pp. 1499-1510. DOI: 10.1029/95RS01826.

Effects of Space Weather on Technology Infrastructure. Netherlands, Kluwer Academic Publishers, 2004. 334 p. DOI: $10.1007 / 1-4020-2754-0$. (NATO Science Series II: Mathematics, Physics and Chemistry, vol. 176).

Erinmez I.A., Kappenman J.G., Radasky W.A. Management of the geomagnetically induced current risks on the national grid company's electric power transmission system. J. Atmos. Solar-Terr. Phys. 2002, vol. 64, no. 5-6, pp. 743-756. DOI: 10.1016/S1364-6826(02)00036-6.

Fel'dshtejn Ya.I., Vorob'ev V.G., Zverev V.L. Planetary patterns of auroras. The results of the International Geophysical Year (Review). Geomagnetizm i aeronomiya [Geomagnetism and Aeronomy]. 2010, vol. 50, no. 4, pp.435-458. (In Russian).

Fridman S.V., Nickisch L.J., Hausman M., Zunich G. Assimilative model for ionospheric dynamics employing delay, Doppler, and direction of arrival measurements from multiple HF channels. Radio Sci. 2016, vol. 51, no. 3, pp. 176-183. DOI: 10.1002/2015rs005890.

Gauld J.K., Yeoman T.K., Davies J.A., et al. SuperDARN radar HF propagation and absorption response to the substorm expansion phase. Ann. Geophys. 2002, vol. 20, pp. 16311645. DOI: 10.5194/angeo-20-1631-2002.

Ghasemi A., Abedi A., Ghasemi F. Propagation Engineering in Radio Links Design. New York, Springer-Verlag, 2013, 549 p. DOI: 10.1007/978-1-4614-5314-7.

Gherm V.E., Zernov N.N., Strangeways H.J. Effects of diffraction by ionospheric electron density irregularities on the range error in GNSS dual-frequency positioning and phase decorrelation. Radio Sci. 2011, vol. 46, no. 3, RS3002. DOI: 10.1029/2010rs004624.

Gillies R.G., Hussey G.C., Sofko G.J., et al. Improvement of HF coherent radar line-of-sight velocities by estimating the refractive index in the scattering volume using radar frequency shifting. J. Geophys. Res. 2011, vol. 116, A01302. DOI: 10.1029/ 2010JA016043.

Ginzburg V.L. Rasprostranenie electromagnitnyhk voln v plazme [Propagation of Electromagnetic Waves in Plasma]. Moscow, Fizmatizdat Publ., 1960. 550 p. (In Russian).

Gombosi T.I., Baker D.N., Balogh A., Erickson P.J., Huba J.D., Lanzerotti L.J. Anthropogenic Space Weather. Space Sci. Rev. 2017, 55 p. DOI: 10.1007/s11214-017-0357-5.

Goodman J.M. Electron content inhomogeneities in the lower ionosphere. J. Geophys. Res. 1967. vol. 72, no. 21, pp. 55425546. DOI: 10.1029/JZ072i021p05542.

Goodman J.M. HF Communications: Science \& Technology. New York, Van Nostrand Reinhold Publ., 1991, 631 p.

Goodman J.M. Space Weather \& Telecommunications. Springer US, 2005, 382 p. DOI: 10.1007/ b102193.
Goodman J.M., Aarons J. 1990, Ionospheric effects on modern electronic systems. Proc. IEEE. vol. 78, no. 3, pp. 512-528. DOI: $10.1109 / 5.52228$.

Groves K.M., Basu S., Weber E.J., et al. Equatorial scintillation and systems support. Radio Sci. 1997, vol. 32, no. 5, pp. 2047-2064. DOI: 10.1029/97rs00836.

Haldoupis C.I. A review on radio studies of auroral Eregion ionospheric irregularities. Ann. Geophys. 1989, vol. 7, pp. 239-258.

Headrick J.M. Looking over the horizon. IEEE Spectrum. 1990, vol. 27, pp. 36-39. DOI: 10.1109/6.58421.

Headrick J.M., Skolnik M. Over the horizon radar in the HF band. Proc. IEEE. 1974, vol. 62, no.6, pp. 664-673. DOI: 10.1109/PROC.1974.9506.

Hernández-Pajares M., Wielgosz P., Paziewski J., Krypiak-Gregorczyk A., Krukowska M., Stepniak K., Kaplon J., Hadas T., Sosnica K., Bosy J., Orus-Perez R., Monte-Moreno E., Yang H., Garcia-Rigo A., Olivares-Pulido G. Direct MSTID mitigation in precise GPS processing. Radio Sci. 2017, 2016RS006159. DOI: 10.1002/2016rs006159.

Hunsucker R.D. Atmospheric gravity waves generated in the high-latitude ionosphere: A review. Rev. Geophys. 1982, vol. 20, no. 2, pp. 293-315. DOI: 10.1029/RG020i002p00293.

Ionizing Radiation Effects in Electronics: From Memories to Imagers. CRC Press, 2015. 394 p.

Ivanov V.A., Kurkin V.I., Nosov V.E., et al. CHIRP ionosonde and its application in the ionospheric research. Radiophysics and Quantum electronics. 2003, vol. 46, no. 11, pp. 821-851.

Keil W. Radiation Effects on Spacecraft and Countermeasures, Selected Cases. Space Weather: Research Towards Applications in Europe. Dordrecht: Springer, 2007, pp. 231-240. DOI: 10.1007/1-4020-5446-7 21.

Kim H., Clauer C.R., Deshpande K., et al. Ionospheric irregularities during a substorm event: Observations of ULF pulsations and GPS scintillations. J. Atmos. Solar-Terr. Phys. 2014, vol. 114, pp. 1-8. DOI: 10.1016/j.jastp. 2014.03.006.

Klobuchar J.A. A First-Order, Worldwide, Ionospheric Time Delay Algorithm. AFCRL-TR-75-0502. 1975, ADA018862, 26 p.

Klobuchar J.A., Anderson D.N., Bishop G.J., Doherty P.H. Measurements of transionospheric propagation parameters in the polar cap ionosphere. AFGL-TR-87-1021. 1987, no. 973, 12 p.

Knight P. Medium frequency propagation: A survey. $B B C$ RD 1983/5 (RA210). 1983, 20 p.

Kravtsov Yu.A., Orlov Yu.A. Geometrichkaya optika neodnorodnykh sred [Geometrical Optics of Inhomogeneous Media]. Moscow, Nauka Publ., 1980, 304 p. (In Russian).

Kurkin V.I., Orlov I.I., Popov V.N. Metod normal'nykh voln v probleme korotkovolnovoi radiosvyazi [Normal Mode Method in a Problem of Short-Wave Radio Communication]. Moscow, Nauka Publ., 1981, 124 p. (In Russian).

Kutelev K.A., Berngardt O.I. Simulation SuperDARN radar ground backscatter signal in the presence of moving midscale inhomogenity during the fall of Chelyabinsk meteorite. Solnechno-zemnaja fizika [Solar-Terr. Phys.]. 2013, vol. 24, pp. 15-26. (In Russian)

Kutiev I., Tsagouri I., Perrone L., et al. Solar activity impact on the Earth's upper atmosphere. J. Space Weather and Space 
Climate. 2013, vol. 3, no. 27, A06. DOI: 10.1051/swsc/2013028.

Landeau T., Gauthier F., Ruelle N. Further improvements to the inversion of the elevation-scan backscatter sounding data. J. Atmos. Solar-Terr. Phys. 1997, vol. 59, no. 1, pp. 125-138.

Lee J. Radio emissions from solar active regions. Space Sci. Rev. 2007, vol. 133, pp. 73-102. DOI: $10.1007 /$ s11214007-9206-2.

Leonovich A.S., Mazur V.A. Eigen ultra-low magnetoacoustic oscillations of the near plasma sheet. Kosmicheskie issledovaniya [Space Res.]. 2008, vol. 46, no. 4, pp. 336-343. (In Russian).

Liu H., Wang W., Richmond A.D., Roble R.G. Ionospheric variability due to planetary waves and tides for solar minimum conditions. J. Geophys. Res.: Space Phys. 2010, vol. 115, no. A6, A00G01. DOI: 10.1029/2009JA015188.

Liu H., Jin H., Miyoshi Y., Fujiwara H., Shinagawa H. Upper atmosphere response to stratosphere sudden warming: local time and height dependence simulated by GAIA model. Geophys. Res. Lett. 2013, vol. 40, no. 3, pp. 635-640. DOI: 10.1002/grl.50146.

Lognonné P., Clévédé E., Kanamori H. Computation of seismograms and atmospheric oscillations by normal-mode summation for a spherical Earth model with realistic atmosphere. Geophys. J. Intern. 1998, vol. 135, no. 2, pp. 388-406. DOI: 10.1046/j.1365-246x.1998.00665.x.

Lotóaniu T.M., Singer H.J., Rodriguez J.V., et al. Space weather conditions during the Galaxy 15 spacecraft anomaly. Space Weather. 2015, vol. 13, no. 8, pp. 484-502. DOI: 10.1002/ 2015 SW001239.

Love J.J., Coïsson P. The geomagnetic blitz of September 1941. Eos. Earth \& Space Science News. 2016, vol. 97, pp. 18-22. DOI: 10.1029/2016EO059319.

Love J.J., Pulkkinen A., Bedrosian P.A., et al. Geoelectric hazard maps for the continental United States. Geophys. Res. Lett. 2016, vol. 43, no. 18, pp. 9415-9424. DOI: 10.1002/ 2016 GL070469.

Machol J.L., Green J.C., Redmon R.J., Viereck R.A., Newell P.T. Evaluation of OVATION as a forecast model for visible aurorae. Space Weather. 2012, vol. 10, no. 3, S03005. DOI: 10.1029/2011SW000746.

Mager P.N., Berngardt O.I., Klimushkin D.Yu., et al. First results of the high-resolution multibeam ULF wave experiment at the Ekaterinburg SuperDARN radar: ionospheric signatures of coupled poloidal Alfven and drift-compressional modes. J. Atmos. Solar-Terr. Phys. 2015, vol. 130-131, pp. 112-126. DOI: 10.1016/j.jastp.2015.05.017.

Marshall R.A., Smith E.A., Francis M.J., et al. A preliminary risk assessment of the Australian region. Space Weather. 2011, vol. 9, no. 10, S10004. DOI: 10.1029/2011 SW000685.

Mikhailov A.V., Perrone L. Geomagnetic control of the midlatitude daytime $f_{\mathrm{o}} \mathrm{F} 1$ and $f_{\mathrm{o}} \mathrm{F} 2$ long-term variations: physical interpretation using European observations. J. Geophys. Res.: Space Phys. 2016, vol. 121, no. 7, pp. 7193-7203. DOI: 10.1002/ $2016 \mathrm{ja} 022716$.

Mullen J.P., MacKenzie E., Basu S., Whitney H. $\mathrm{UHF} / \mathrm{GHz}$ scintillation observed at Ascension Island from 1980 through 1982. Radio Sci. 1985, vol. 20, no. 3, pp. 357365. DOI: 10.1029/RS020i003p00357.

National Space Weather Action Plan. National Science and Technology Council, 2015. $42 \mathrm{p}$.

National Space Weather Strategy. National Science and Technology Council, 2015. 18 p.

Obama B. Executive Order: Coordinating Efforts to Prepare the Nation for Space Weather Events. 2016. URL: https://www.whitehouse.gov/the-press-office/

2016/10/13/executive-order-coordinating-efforts-prepare-nationspace-weather-events (accessed December 12, 2016).

Odstrcil D. Modeling 3-D solar wind structure. Adv. Space Res. 2003, vol. 32, no. 4, pp. 497-506. DOI: http://dx.doi.org/10.1016/S0273-1177(03)00332-6.

Ogawa T., Nishitani N., Tsugawa T., Shiokawa K. Giant ionospheric disturbances observed with the SuperDARN Hokkaido HF radar and GPS network after the 2011 Tohoku earthquake. Earth, Planets and Space. 2012, vol. 64, pp. 1295-1307. DOI: 10.5047/eps.2012.08.001.

Oinats A.V., Nishitani N., Ponomarenko P., Berngardt O.I., Ratovsky K.G. Statistical characteristics of the medium-scale travel ionospheric disturbances revealed from the Hokkaido East and Ekaterinburg HF radar data. Earth, Planets and Space. 2016, vol. 68, iss. 1, 8. DOI: 10.1186/s40623-016-0390-8.

Pappert R.A. Effects of a large patch of sporadic E on the nighttime propagation at lower ELF. J. Atmos. Solar-Terr. Phys. 1980, vol. 42, no. 5, pp. 417-425. DOI: 10.1016/00219169(80)90001-X.

Pappert R.A., Moler W.F. A theoretical study of ELF normal mode reflection and absorption produced by nighttime ionospheres. J. Atmos. Solar-Terr. Phys. 1978, vol. 40, no. 9, pp. 1031-1045. DOI: 10.1016/0021-9169(78)90008-9.

Patra A.K., Srinivasulu P., Pavan Chaitanya P.P. First results on low-latitude E- and F-region irregularities obtained using the Gadanki Ionospheric Radar Interferometer (GIRI). J. Geophys. Res.: Space Phys. 2014, vol. 119, no. 12, pp. 10276 10293. DOI:10.1002/2014ja020604.

Perrone L., Alfonsi L., Romano V., de Franceschi G. Polar cap absorption events of November 2001 at Terra Nova Bay, Antarctica. Ann. Geophys. 2004, vol. 22, no. 5, pp. 16331648. DOI: 10.5194/angeo-221633-2004.

Pirjola R. Geomagnetically induced currents during magnetic storms. IEEE Trans. Plasma Sci. 2000, vol. 28, no. 6, pp. 1867-1873. DOI: 10.1109/27.902215.

Principles of Modern Radar: Basic Principles. Scitech Publishing, 2010. 960 p. DOI: 10.1049/SBRA021E.

Priyadarshi S. A review of ionospheric scintillation models. Surveys in Geophysics. 2015, vol. 36, no. 2, pp. 295-324. DOI: 10.1007/s10712-015-9319-1.

Pulkkinen A., Lindahl S., Viljanen A., Pirjola R. Geomagnetic storm of 29-31 October 2003: geomagnetically induced currents and their relation to problems in the Swedish high-voltage power transmission system. Space Weather. 2005, vol. 3, no. 8, S08C03. DOI: 10.1029/200 4SW000123.

Radicella S.M., Leitinger R. The evolution of the DGR approach to model electron density profiles. Adv. Space Res. 2001, vol. 27, no. 1, pp. 35-40. DOI: 10.1016/S0273-1177(00) 00138-1.

Reinisch B.W., Haines D.M., Bibl K., et al. Ionospheric sounding in support of over-the-horizon radar. Radio Sci. 1997, vol. 32, no. 4, pp. 1681-1694. DOI: 10.1029/97rs00841.

Rino C.L., Matthews S.J. On the morphology of the auro- 
ral zone radio wave scintillation. J. Geophys. Res. 1980, vol. 85, no. A8, pp. 4139-4151. DOI: 10.1029/JA085iA08p04139.

Rogov D.D., Moskaleva E.V., Zaalov N.Y. Modeling of high frequency radio wave absorption on oblique soundings during a solar X-ray flare. Adv. Space Res. 2015, vol. 55, no. 2, pp. 597-604. DOI: 10.1016/j.asr.2014.11.001.

Rostoker G. Geomagnetic indices. Rev. Geophys. 1972, vol. 10, no. 4, pp. 935-950. DOI: 10.1029/RG010i004p00935.

Schumann W.O. Über die strahlungslosen Eigenschwingungen einer leitenden Kugel, die von einer Luftschicht und einer Ionosphärenhülle umgeben ist. Zeitschrift für Naturforschung A. 1952, vol. 7, iss. 2, pp. 149-154. DOI: 10.1515/ zna-1952-0202. (In German).

Settimi A., Ippolito A., Cesaroni C., Scotto C. Scientific review on the ionospheric absorption and research prospects of a complex eikonal model for one-layer ionosphere. Intern. $J$. Geophys. 2014, vol. 2014. DOI: 10.1155/ 2014/657434.

Settimi A., Pietrella M., Pezzopane M., Bianchi C. The IONORT-ISP-WC system: Inclusion of an electron collision frequency model for the D-layer. Adv. Space Res. 2015, vol. 55, no. 8, pp. 2114-2123. DOI: 10.1016/j.asr. 2014.07.040.

Severe Space Weather Events - Understanding Societal and Economic Impacts: A Workshop Report. Washington, The National Academies Press, 2008, 144 p. DOI: 10.17226/12507.

Shibasaki K., Alissandrakis C.E., Pohjolainen S. Radio emission of the quiet Sun and active regions (invited Review). Solar Phys. 2011, vol. 273, pp. 309-337. DOI: 10.1007/ s11207-011-9788-4.

Solar and Space Physics: A Science for a Technological Society. Washington, The National Academies Press, 2013, 446 p. DOI: $10.17226 / 13060$.

Solar and Space Weather Radiophysics - Current Status and Future Developments. Dordrecht, Kluwer Academic Publishers, 2004, 424 p. DOI: 10.1007/1-4020-2814-8. (Astrophys. Space Sci. Library, vol. 314).

Sonnenschein E., Censor D., Rutkevich I., Bennett J.A. Ray trajectories in an absorbing ionosphere. J. Atmos. Solar-Terr. Phys. 1997, vol. 59, no. 16, pp. 2101-2110. DOI: 10.1016/s13646826(97)00035-7.

Space Weather - Effects on Technology. Canadian Space Weather Forecast Centre Publ., 2012, 3 p.

Space Weather: Research Towards Applications in Europe. Dordrecht, Springer, 2007. 332 p. DOI: 10.1007/1-4020-5446-7.

Stocker A.J., Arnold N.F., Jones T.B. The synthesis of travelling ionospheric disturbance (TID) signatures in HF radar observations using ray tracing. Ann. Geophys. 2000, vol. 18, no. 1, pp. 56-64. DOI: 10.1007/s00585-000-0056-4.

Sverdlov Ju.L. Morfologiya radioavrory [Morphology of Radioaurora]. Leningrad, Nauka Publ., 1982, 160 p. (In Russian).

The Sun to the Earth - and Beyond: A Decadal Research Strategy in Solar and Space Physics. Washington, The National Academies Press, 2003, p. DOI: 10.17226/10477.

Thomson A.W.P., Dawson E.B., Reay S.J. Quantifying extreme behavior in geomagnetic activity. Space Weather. 2011, vol. 9, no. 10, S10001. DOI: 10.1029/2011SW000696.

Troshichev O., Janzhura A., Stauning P. Unified PCN and PCS indices: method of calculation, physical sense, and dependence on the IMF azimuthal and northward components. J. Ge- ophys. Res.: Space Phys. 2006, vol. 111, no. A5, A05208. DOI: 10.1029/2005JA011402.

Tsunoda R.T. High-latitude F region irregularities: a review and synthesis. Rev. Geophys. 1988, vol. 26, no. 4, pp. 719-760. DOI: $10.1029 /$ RG026i004p00719.

Tsunoda R.T., Maruyama T., Tsugawa T., Yokoyama T., Ishii M., Nguyen T.T., Ogawa T., Nishioka M. Off-greatcircle paths in transequatorial propagation 2. Non-magneticfield-aligned reflections. J. Geophys. Res.: Space Phys. 2016, vol. 121 , iss. 11 , pp. 11,176-11,190. DOI: 10.1002/2016ja 022404.

Uryadov V.P., Vertogradov G.G., Vertogradova E.G. SpreadF radar observations in the midlatitude ionosphere using an Ionosonde-Radiodirection Finder. Radiophys. Quantum Electronics. 2013, vol. 56, no. 1, pp. 1-11. DOI: 10.1007/s11141-0139411-7.

Vilensky I.M., Yampol'sky V.S., Tsydypov C.T. Rasprostranenie srednikh radiovoln $v$ ionosfere [Propagation of Medium Radio Waves in the Ionosphere]. Nauka Publ., 1983, 116 p. (In Russian).

Wang J.C.H. A sky-wave propagation study in the preparation for the 1605-1705 kHz broadcasting conference. IEEE Trans. on Broadcasting. 1985, vol. BC-31, no. 1, pp. 10-17. DOI: 10.1109/ TBC.1985.266546.

Warrington E.M., Stocker A.J., Siddle D.R., et al. Near real-time input to a propagation model for nowcasting of $\mathrm{HF}$ communications with aircraft on polar routes. Radio Sci. 2016, vol. 51, no. 7, pp. 1048-1059. DOI: 10.1002/2015rs005880.

Weber E.J., Tsunoda R.T., Buchau J., et al. Coordinated measurements of auroral zone enchancements. J. Geophys. Res. 1985, vol. 90, no. A7, pp. 6497-9513. DOI: 10.1029/ JA090iA 07p06497.

Wernik A.W., Secan J.A., Fremouw E.J. Ionospheric irregularities and scintillation. Adv. Space Res. 2003, vol. 31, no. 4, pp. 971-981. DOI:10.1016/s02731177(02)00795-0.

Whiteson D., Mulhearn M., Shimmin C., et al. Observing ultra-high energy cosmic rays with smartphones. LANL ArXiv. 2014. arXiv:1410.2895.

Wikipedia. List of Citizen science projects. 2016. URL: https:/en. wikipedia.org/wiki/List_of_citizen_science_projects (accessed December 12, 2016).

Xiong C., Stolle C., Lühr H. The Swarm satellite loss of GPS signal and its relation to ionospheric plasma irregularities. Space Weather. 2016, vol. 14, no. 8, pp. 563-577. DOI: 10.1002/2016SW001439.

Zolesi B., Cander L.R. Ionospheric Prediction and Forecasting. Berlin, Heidelberg Springer Geophysics, 2014, 252 p. DOI: $10.1007 / 978-3-642-38430-1$.

URL: http://www.swpc.noaa.gov/products/goes-x-ray-flux (accessed December 12, 2016).

URL: http://www.swpc.noaa.gov (accessed December 12, 2016).

How to cite this article

Berngardt O.I. Space weather impact on radio device operation. Solar-Terrestrial Physics. 2017. Vol. 3, iss. 3. P. 37-53. DOI: 10.12737/ stp-33201705 